\title{
AN INFLUENCE OF A NON-PRISMATIC ROD AS A PART OF A GEOMETRICALLY NONLINEAR SYSTEM UNDER GENERALIZED LOAD WITH A FORCE DIRECTED TOWARDS THE POSITIVE POLE ON THE VALUE OF THE BIFURCATION LOAD
}

\author{
J. Szmidla*, A. Jurczyńska**
}

\begin{abstract}
The article presents the theoretical considerations and results of the numerical computations regarding the issue of stability of the geometrically nonlinear column with the non-prismatic rod under the generalized load with the force directed towards the positive pole (case of L. Tomski's load). The boundary problem was formulated on the basis of minimum potential energy principle and solved using the small parameter of amplitude method (perturbation method). It was proved that appropriate selection of the shape of the rod of the cross-section variable along the axis results in an increase in the value of the bifurcation load of the column compared to the reference system (built of prismatic rods of the same volume) and thus have an influence on the range of local loss of rectilinear form of static equilibrium.
\end{abstract}

Keywords: stability, geometrically nonlinear, specific load, nonprismatic, perturbation method

\section{Introduction}

Taking into account the nonlinearity commonly found in the real objects, research on the nonlinear systems are important in mechanics. In addition, considering the widespread use of the slender beams, rods and columns in the mechanical constructions or civil engineering, a particular attention should be paid to the issues of their free vibrations and stability. Article (Uzny et all., 2016) concerns free vibrations of a partially tensioned column. The problem of the nonlinearity of the slender system was also the subject of the work (Akour, 2012) where support by the elastic foundation was analyzed. In the work (Sochacki, 2015) the analysis of the damped vibrations in the hydraulic cylinder was presented. The subject of the paper (Uzny and Sokól, 2015) was an influence of the theory adopted in the mathematical model on the natural frequency.

\section{Physical Model}

The slender geometrically nonlinear system $\mathrm{N}$ built of three prismatic rods, the free ends of which are connected by the element of mass $m$ ensuring the equality of deflection and angles of deflection (see fig. 1c), is considered in this paper. Taking into account the condition of constant total flexural stiffness, the column is described by the coefficient of flexural stiffness distribution $\mu$ that is given in the following form:

$$
\mu=\frac{(E J)_{I I I}}{(E J)_{I}+(E J)_{I I}}
$$

Associate Prof. Janusz Szmidla, PhD. Eng.: Institute of Mechanics and Machinery Design Fundamentals, Częstochowa University of Technology,Dąbrowskiego 73; 42-201 Częstochowa; PL, j.szmidla@imipkm.pcz.pl

** Anna Jurczyńska, MSc. Eng.:Institute of Mechanics and Machinery Design Fundamentals, Częstochowa University of Technology,Dąbrowskiego 73; 42-201, Częstochowa; PL, a.jurczynska@imipkm.pcz.pl 


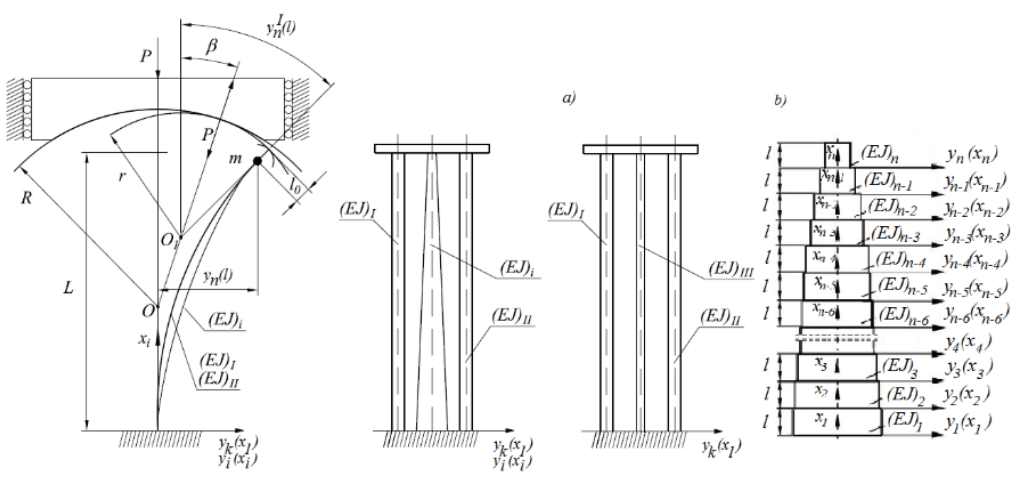

Fig. 1: Physical model of the geometrically nonlinear columnwith a nonprismatic rod NNsubjected to the generalized load with a force directed towards the positive pole a) comparative system:

geometrically nonlinear column $\mathrm{N}$ b) model of the rod of variable cross-section.

In the target system of the geometrically nonlinear column with the rod of the cross-section variable along the axis NN (fig. 1a) the internal rod of the structure $\mathrm{N}$ was replaced by the non-prismatic rod. The variable cross-section was modelled by the dividing the bar into $n$ prismatic segments of constant length and variable width described by the approximating functions, while maintaining the condition of constant total volume resulting from the value of the coefficient $\mu$. The carried out consideration and simulations were limited to the shape approximation by means of the polynomial of degree 1 and 2 . In order to determine the ranges of the local and global loss of the rectilinear form of the static equilibrium, the second comparative system is also considered - geometrically linear column L built of two external prismatic rods of the geometrically nonlinear structures.

All the analyzed columns are subjected to the generalized load with a force directed towards the specific load (case of the specific load defined by the L. Tomski). The loading is implemented through the structure that is made of the elements of a circular outlines: the loading head of the radius $R$ and the receiving head of the radius $r$. For structural reasons, the considerations also include the rigid element of the length $l_{0}$ enabling the column to be properly mounted.

\section{Mathematical Model}

On the basis of the physical model of the geometrically nonlinear column with the rod of variable crosssection, the potential energy is defined as a sum of the bending elasticity energy of the particular rods and segments of the systemand the component resulting from the adopted external load:

$$
\begin{gathered}
V=\frac{1}{2} \sum_{k=I}^{I I}(E J)_{k} \int_{0}^{L}\left[y_{k}{ }^{I I}\left(x_{1}\right)\right]^{2} d x_{1}+\frac{1}{2} \sum_{i=1}^{n}(E J)_{i} \int_{0}^{l}\left[y_{i}^{I I}\left(x_{i}\right)\right]^{2} d x_{i}+ \\
-\frac{1}{2} P l_{0}\left[y_{n}^{I}(l)\right]^{2}+\frac{1}{2}\left(\sum_{k=I}^{I I}(E A)_{k} \int_{0}^{L}\left[u_{k}^{I}\left(x_{1}\right)+\frac{1}{2}\left(y_{k}{ }^{I}\left(x_{1}\right)\right)^{2}\right]^{2} d x_{1}\right)+ \\
+\frac{1}{2}\left(\sum_{i=1}^{n}(E A)_{i} \int_{0}^{l}\left[u_{i}^{I}\left(x_{i}\right)+\frac{1}{2}\left(y_{i}^{I}\left(x_{i}\right)\right)^{2}\right]^{2} d x_{i}\right)+P u_{I}(L)+P \frac{r}{2}\left[\left[y_{n}{ }^{I}(l)\right]^{2}-\beta^{2}\right]
\end{gathered}
$$

The boundary problem was formulated on the basis of minimum potential energy principle (static criterion of the stability loss):

$$
\delta V=0
$$

After substituting the equation (2) into the formula (3) and after performing the variational calculus, the following relations were obtained:

- the differential equations of displacements in the transverse direction:

$$
\begin{aligned}
& (E J)_{k} y_{k}^{I V}\left(x_{1}\right)+S_{k} y_{k}^{I I}\left(x_{1}\right)=0, \\
& (E J)_{i} y_{i}^{I V}\left(x_{i}\right)+S_{I I I} y_{i}^{I I}\left(x_{i}\right)=0,
\end{aligned}
$$


- the differential equations of displacements in the longitudinal direction:

$$
\begin{aligned}
& \frac{d}{d x_{1}}\left[u_{k}^{I}\left(x_{1}\right)+\frac{1}{2}\left(y_{k}^{I}\left(x_{1}\right)\right)^{2}\right]=0, \quad k=I, I I \\
& \frac{d}{d x_{i}}\left[u_{i}^{I}\left(x_{i}\right)+\frac{1}{2}\left(y_{i}^{I}\left(x_{i}\right)\right)^{2}\right]=0, \quad i=1,2, \ldots ., n
\end{aligned}
$$

where $S_{k}, S_{I I I}$ are the internal forces in the particular rods of the system, determined taking into account Hooke's law:

$$
S_{I I I}=\frac{P}{\frac{(E A)_{1}}{n}\left(\sum_{i=1}^{n} \frac{1}{(E A)_{i}}\right)+1}, \quad S_{I}=S_{I I}, \quad \sum_{k=I}^{I I I} S_{k}=P
$$

The boundary conditions as well as the continuity conditions are written in the following form:

$$
\begin{gathered}
y_{I}(0)=y_{I I}(0)=y_{1}(0)=0, \quad y_{I}(L)=y_{I I}(L)=y_{n}(l) \\
u_{I}(0)=u_{I I}(0)=u_{1}(0)=0, \quad u_{I}(L)=u_{I I}(L)=u_{n}(l) \\
y_{I}{ }^{I}(0)=y_{I I}{ }^{I}(0)=y_{1}{ }^{I}(0)=0, \quad y_{I}{ }^{I}(L)=y_{I I}{ }^{I}(L)=y_{n}{ }^{I}(l) \\
\sum_{k=I}^{I I}(E J)_{k} y_{k}{ }^{I I}(L)+(E J)_{n} y_{n}{ }^{I I}(l)+\left(r-l_{0}\right)\left\{\frac{P}{R-r}\left[\left(R-l_{0}\right) y_{I}{ }^{I}(L)-y_{I}(L)\right]\right\}=0 \\
\sum_{k=I}^{I I}(E J)_{k} y_{k}{ }^{I I I}(L)+(E J)_{n} y_{n}{ }^{I I I}(l)+\frac{P}{R-r}\left[\left(R-l_{0}\right) y_{I}{ }^{I}(L)-y_{I}(L)\right]-m \omega^{2} y_{I}(L)=0 \\
y_{j}(l)=y_{j+1}(0), \quad u_{j}(l)=u_{j+1}(0), \quad y_{j}{ }^{I}(l)=y_{j+1}{ }^{I}(0), \\
(E J)_{j} y_{j}{ }^{I I}(l)=(E J)_{j+1} y_{j+1}{ }^{I I}(0), \\
(E J)_{j} y_{j}{ }^{I I I}(l)=(E J)_{j+1} y_{j+1}{ }^{I I I}(0),
\end{gathered}
$$

The solution of the boundary problem was obtained using the small parameter of the amplitude method (perturbation method). Supplementing the conditions (7-14) with the solutions of the differential equations (4) makes it possible to obtain a system of homogeneous equations and a transcendent equation for the value of bifurcation load.

\section{Results of the numerical computations}

In order to compare the obtained results, the following dimensionless parameters are introduced:

$$
p^{*}=\frac{p}{L}, \quad q^{*}=\frac{q}{L}, \quad Z^{*}=\frac{b_{1}-b_{n}}{L} \cdot 100 \%, \Delta \mathrm{r}=\frac{\mathrm{R}-\mathrm{r}}{\mathrm{L}}, R_{i}^{*}=\frac{R_{i}-l_{0}}{L}, \quad \lambda_{b}=\frac{P_{b} \cdot L^{2}}{\sum_{k=I}^{I I I}(E J)_{k}}(15 \mathrm{a}-\mathrm{f})
$$

Figure 1 shows the values of the bifurcation load of the columns: geometrically nonlinear $\mathrm{N}$ and geometrically linear $\mathrm{L}$ as a function of the coefficient of flexural stiffness distribution $\mu$. In the initial range of the coefficient $\mu$ the linear column built of two rods is characterized by the larger bifurcation load than the geometrically nonlinear column constructed of three bars. This area is called the area of the local loss of the rectilinear form of the static equilibrium. Above $\mu_{g r}$ value the bifurcation load of the nonlinear system is larger than the bifurcation load of the linear column and the structure is in the area of the global loss of the rectilinear form of the static equilibrium. 


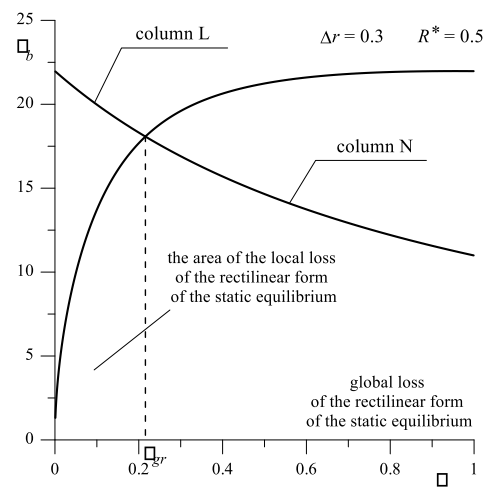

Fig. 2: The comparison of the bifurcation load of the geometrically linear $L$ and geometrically nonlinear $N$ column as a function of the coefficient of the flexural stiffness distribution $\mu$.
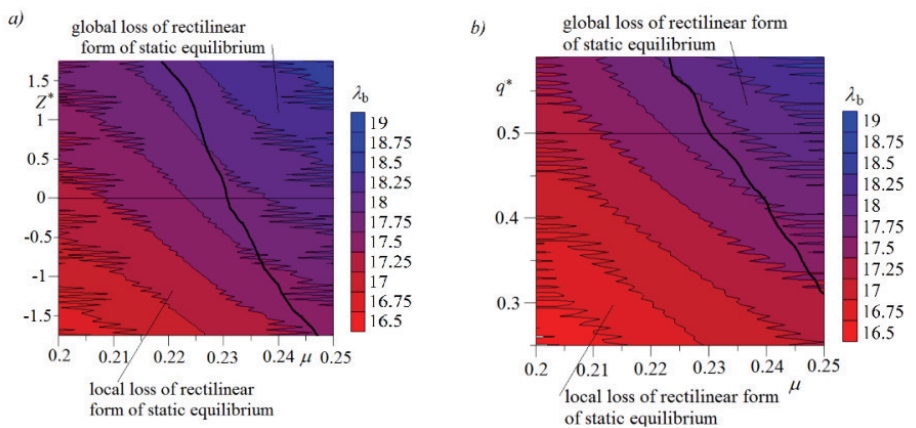

Fig. 3: The value of the bifurcation load and the ranges of the local loss of rectilinear form of the static equilibrium of the geometrically nonlinear column $N N$ with non-prismatic rod approximated a) by the linear function $\left(\Delta r=0.2, R^{*}=0.4\right)$ b) by the quadratic function $\left(\Delta r=0.4, R^{*}=0.6\right)$.

The values of the bifurcation load presented in the figure 3 refer to the geometrically nonlinear column with the non-prismatic rod. The shown data illustrate the influence of the values of the coefficient defining the approximating functions on the values of bifurcation load as well as ranges of the local and global loss of the rectilinear form of the static equilibrium.

\section{Conclusions}

Geometrically systems are characterized by two forms of the static equilibrium. On the basis of the presented results of the numerical computations it was proved that the appropriate selection of the shape approximating functions of the non-prismatic rod as a structural part of the geometrically nonlinear system may cause an increase in the value of the bifurcation load and thus reduce the range of the local loss of the rectilinear form of the static equilibrium. The next stage of the research should be the experimental verification of the assumptions adopted in the model.

\section{Acknowledgement}

The study has been carried out within statutory research BS/PB 1-101-3020/17/P of the Institute of Mechanics and Machinery Design Fundamentals of Częstochowa University of Technology.

\section{References}

Akour, S. N. (2012) Parametric Study of Nonlinear Beam Vibration Resting on Linear Elastic Foundation, Journal of Mechanical Engineering and Automation, vol. 2 (6), pp. 114-134.

Sochacki, W. (2015) Modelling and analysis of damped vibration in hydraulic cylinder, Mathematical and Computer Modelling of Dynamical Systems, vol. 21(1), pp. 23-37.

Uzny, S., Sokół, K, Osadnik, M. (2016) Influence of amplitude on free vibration frequency of a partially tensioned column, Engineering Mechanics, pp.562-565.

Uzny, S., Sokół, K. (2015) The Bernoulli-Euler and Timoshenko Theories in the context of research on the characteristic curves of column with different boundary conditions, AIP Conference Proceedings, 1648, DOI: 10.1063/1.4913091. 